\title{
A neutral atom quantum register
}

\author{
D. Schrader, I. Dotsenko, M. Khudaverdyan, Y. Miroshnychenko, A. Rauschenbeutel, and D. Meschede* \\ Institut für Angewandte Physik, Universität Bonn, Wegelerstr. 8, D-53115 Bonn, Germany
}

(Dated: October 17, 2018)

\begin{abstract}
We demonstrate the realization of a quantum register using a string of single neutral atoms which are trapped in an optical dipole trap. The atoms are selectively and coherently manipulated in a magnetic field gradient using microwave radiation. Our addressing scheme operates with a high spatial resolution and qubit rotations on individual atoms are performed with $99 \%$ contrast. In a final read-out operation we analyze each individual atomic state. Finally, we have measured the coherence time and identified the predominant dephasing mechanism for our register.
\end{abstract}

PACS numbers: 03.67.-a, 32.80.Pj, 39.25.+k, 42.50.Vk

Information coded into the quantum states of physical systems (qubits) can be processed according to the laws of quantum mechanics. It has been shown that the quantum concepts of state superposition and entanglement can lead to a dramatic speed up in solving certain classes of computational problems [1, 2]. Over the past decade various quantum computing schemes have been proposed. In a sequential network of quantum logic gates quantum information is processed using discrete one- and two-qubit operations [3]. Another approach is the oneway quantum computer which processes information by performing one-qubit rotations and measurements on an entangled cluster state [4]. All of these schemes rely on the availability of a quantum register, i. e. a well known number of qubits that can be individually addressed and coherently manipulated. There are several physical systems, such as trapped ions [5, 6, 7], nuclear spins in molecules [8], or magnetic flux qubits [9] that can serve as quantum registers.

Neutral atoms exhibit favourable properties for storing and processing quantum information. Their hyperfine ground states are readily prepared in pure quantum states including state superpositions and can be well isolated from their environment. In addition, using laser cooling techniques, countable numbers of neutral atoms can be cooled, captured and transported 10, 11]. The coherence properties of laser trapped atoms have been found to be adequate for storing quantum information 12, 13. Moreover, controlled cold collisions 14] or the exchange of microwave [15] or optical [16, 17] photons in a resonator offer interesting schemes for mediating coherent atom-atom interaction, essential for the realization of quantum logic operations.

In our experiment we use a string of an exactly known number of neutral caesium atoms. The atoms are trapped in the potential wells of a spatially modulated, light induced potential created by a far detuned standing wave dipole trap [10, 18]. They can be optically resolved with an imaging system using an intensified CCD camera (ICCD) 19, 20]. Our experimental setup is schematically depicted in Fig. 1. Two focussed counter-propagating Nd:YAG laser beams at a wavelength of $\lambda=1064 \mathrm{~nm}$

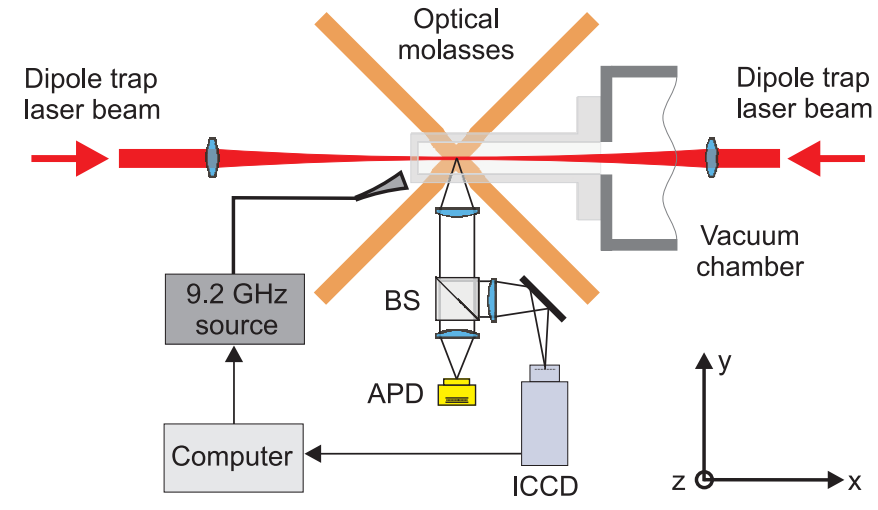

FIG. 1: Scheme of the experimental setup. Two focussed counter-propagating $\mathrm{Nd}$ :YAG laser beams form the dipole trap. We illuminate the trapped atoms by an optical molasses and split the fluorescence light with a beamsplitter (BS) for imaging onto an avalanche photo diode (APD) and an intensified CCD camera (ICCD). Using the information about the atom positions, a computer calculates the corresponding atomic resonance frequencies which are then transmitted to the microwave source.

generate the trapping potential with a depth of up to $2.1 \mathrm{mK}$. This dipole trap is loaded from a high-gradient magneto-optical trap (MOT). We determine the exact number of atoms from the discrete levels of fluorescence of the MOT [21]. The transfer efficiency between the traps is close to $100 \%$. The storage time of $25 \mathrm{~s}$ in the dipole trap is limited by collisions with the background gas. In order to image the atoms, we illuminate them with a red-detuned three-dimensional optical molasses which provides Doppler cooling. The fluorescence light is observed by means of the ICCD with a spatial resolution of $2.7 \mu \mathrm{m}$. Further details of the setup can be found in previous publications 10, 13, 18, 20, 22].

Fig. 2 (a) shows a picture of a string of five trapped atoms. After their transfer from the MOT we let the atoms freely expand along the dipole trap by switching off one of the dipole trap laser beams for $1 \mathrm{~ms}$. Following this expansion the atoms are distributed over an interval of roughly $100 \mu \mathrm{m}$ in the standing wave trap. 


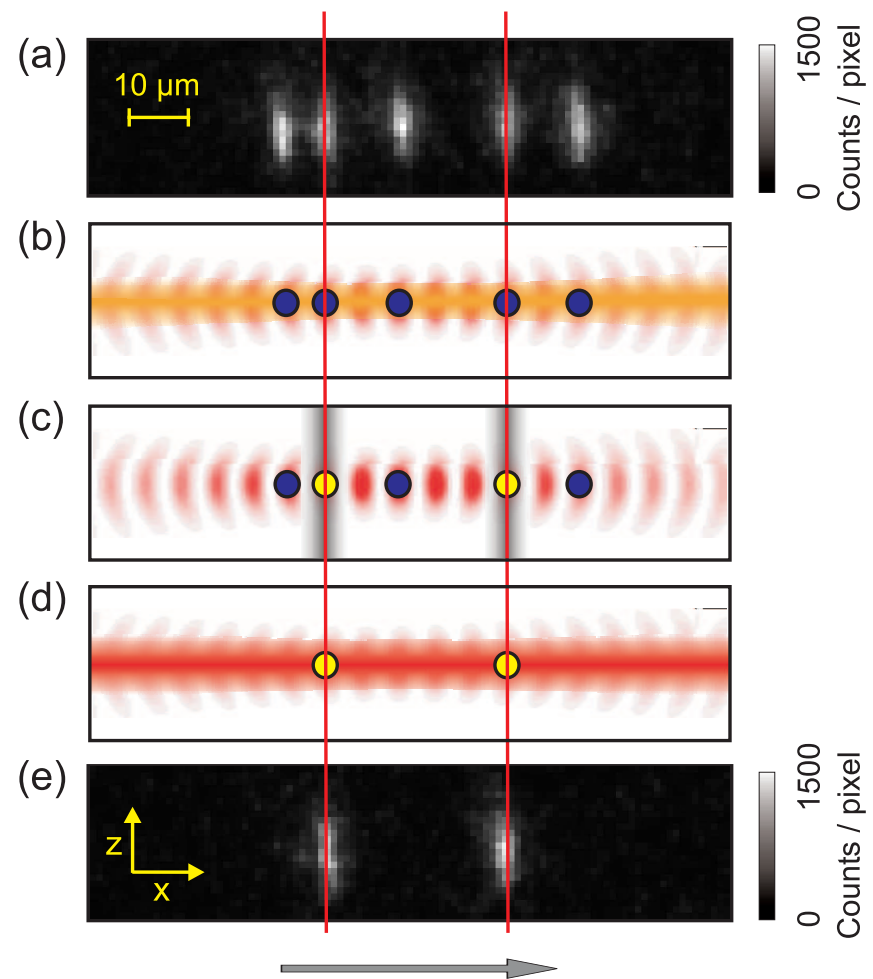

Magnetic field gradient

FIG. 2: 5-atom quantum register. (a) Image of five neutral atoms trapped in separate potential wells of a standing wave dipole trap. The exposure time is $500 \mathrm{~ms}$. One detected photon induces on average 350 counts on the CCD chip. (b) An optical pumping laser initializes the register in state $|00000\rangle$. (c) Two microwave pulses at the resonance frequencies of atoms 2 and 4 perform a spin flip on these atoms to switch to state $|01010\rangle$. The colours indicate the atomic states, blue corresponding to state $|0\rangle$ and yellow to state $|1\rangle$. (d) We state-selectively detect the atoms by applying a pushout laser which removes atoms in state $|0\rangle$ from the trap. (e) A final camera picture confirms the presence of atoms 2 and 4. Note that the spatial period of the schematic potential wells in (b)-(d) is stretched for illustration purposes.

In order to spectroscopically resolve the individual atoms in such a string we apply an inhomogeneous magnetic field which introduces a position dependent hyperfine transition frequency via the Zeeman effect. For experimental simplicity this field is created by means of the coils which also produce the magnetic quadrupole field for the MOT. To achieve the maximum position sensitivity, we work with the stretched $6 S_{1 / 2}$ hyperfine ground states, $\left|F=4, m_{F}=4\right\rangle$ and $\left|F=3, m_{F}=3\right\rangle$, with the quantization axis oriented along the dipole trap axis. These two levels serve as the qubit states in our quantum register and will be denoted $|0\rangle$ and $|1\rangle$, respectively. Our applied magnetic field has the form

$$
\vec{B}(\vec{r})=\left(B_{x}, B_{y}, B_{z}\right)=\left(B_{0}, 0,0\right)-B^{\prime} \cdot(x, y,-2 z) .
$$

A homogeneous offset field $B_{0}=4 \mathrm{G}$ shifts the $|0\rangle \leftrightarrow$ $|1\rangle$ transition frequency by $\nu_{0}=-9.8 \mathrm{MHz}$ with respect to the unperturbed value at $9.2 \mathrm{GHz}$. A gradient field $B^{\prime} \approx 15 \mathrm{G} / \mathrm{cm}$ along the dipole trap yields a positiondependent frequency shift of $\nu^{\prime}=-3.69 \pm 0.04 \mathrm{kHz} / \mu \mathrm{m}$, determined in an initial calibration measurement.

We determine the positions of the atoms along the trap axis by analyzing an ICCD image of the atom string with a fitting routine. From these positions the corresponding atomic resonance frequencies are calculated and sent to the microwave generator. This entire procedure takes about $1 \mathrm{~s}$. We then initialize the register in state $|00000\rangle \equiv|0\rangle_{1}|0\rangle_{2}|0\rangle_{3}|0\rangle_{4}|0\rangle_{5}$, where the subscript denotes the atom number. For this purpose we switch on the magnetic field and optically pump all atoms into state $|0\rangle$ with a $\sigma^{+}$-polarized laser on the $F=4 \leftrightarrow F^{\prime}=4$ transition and a repumping laser on the $F=3 \leftrightarrow F^{\prime}=4$ transition of the $D 2$ line, see Fig. 2 (b).

We now carry out single qubit operations on the initialized register. In this demonstration we switch the register state from $|00000\rangle$ to $|01010\rangle$. For this purpose, we perform spin flips on atoms 2 and 4 by the sequential application of two $\pi$-pulses at their respective frequencies, see Fig. 2 (c). To measure the state of each qubit we switch off the magnetic field and remove all atoms in state $|0\rangle$ from the trap by a state-selective "push-out" laser [13], see Fig. 2 (d). This detection scheme has an efficiency of better than $99 \%$, i. e. less than $1 \%$ of all atoms in state $|1\rangle(|0\rangle)$ are erroneously detected in state $|0\rangle(|1\rangle)$. The presence or absence of each atom in the subsequently taken image therefore reveals its state, $|1\rangle$ or $|0\rangle$, respectively. As expected, atoms 2 and 4 are present in Fig. 2 (e), while atoms 1, 3, and 5 have been removed from the trap.

In order to characterize the performance of our scheme we determine its resolution, i. e. the minimum distance between adjacent atoms necessary for selective addressing. For this purpose, we trap only one atom at a time in our dipole trap and initialize it in state $|0\rangle$. Then we apply a $\pi$-pulse to the atom, with a Gaussian shaped microwave amplitude $A(t)=A_{0} \exp \left(-t^{2} / 2 \sigma_{\tau}^{2}\right)$. The frequency of this microwave pulse is detuned from the atomic resonance frequency at the position of the atom. We record the population transfer from $|0\rangle$ to $|1\rangle$ as a function of this detuning $\delta$ which corresponds to a position offset $\Delta x=\delta / \nu^{\prime}$. For this purpose, we subject the atom to the state-selective push-out laser and reveal its presence or absence through fluorescence detection after retransferring it to the MOT.

The result of this measurement is shown in Fig. 3 (a)(c) for different durations of the microwave pulse. Due to the narrowing Fourier spectrum of the corresponding $\pi$-pulses, the spatial interval of significant population transfer decreases with increasing pulse duration. A pulse of length $2 \sigma_{\tau}=70.7 \mu \mathrm{s}$, see Fig. 3 (c), swaps the state of an atom at one position while an atom trapped 


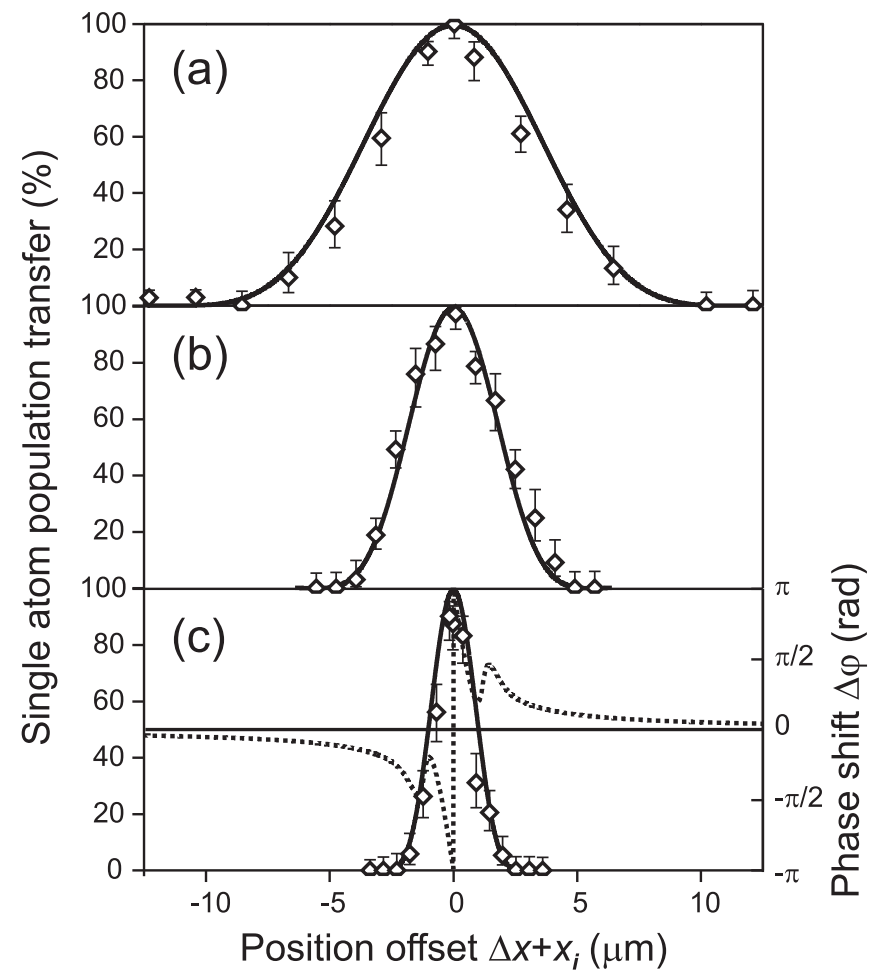

FIG. 3: Measurement of the addressing resolution. The data points show the population transfer efficiency of one atom being exposed to a microwave $\pi$-pulse resonant with a position $\Delta x$ away from the calculated atom position. Each point consists of approximately 40 single atom events. Resonant addressing reveals a spin flip efficiency of close to $100 \%$. For longer pulses $\left(2 \sigma_{\tau}=17.7 \mu \mathrm{s}(\mathrm{a}), 35.4 \mu \mathrm{s}\right.$ (b), and $70.7 \mu \mathrm{s}$ (c)) the spectra become narrower, with an addressing resolution of up to $\sim 2.5 \mu \mathrm{m}$ (c). The center of the spectrum is slightly shifted by $x_{i}$ due to slow drifts of the atomic resonance frequency that occurred during the 10-hour data acquisition time. Here, $x_{a}=-1.0 \mu \mathrm{m}, x_{b}=-2.1 \mu \mathrm{m}$, and $x_{c}=-3.4 \mu \mathrm{m}$. The measured data are in good agreement with a numerical simulation (solid lines). For the parameters in (c) we show the relative phase shift between states $|0\rangle$ and $|1\rangle$, obtained from the same simulation (dotted line).

at a site $2.5 \mu \mathrm{m}$ away remains in its initial state with a probability of $100_{-2.7}^{+0} \%$. The predominant limitation for the addressing resolution are slow drifts of the intensity and the polarization of the dipole trap laser beams which change the atomic resonance frequency by up to $1 \mathrm{kHz} / \mathrm{h}$.

The maximum population transfer for resonant addressing is $98.7_{-3.0}^{+1.1} \%$ for a pulse length of up to $35 \mu \mathrm{s}$. This efficiency includes all experimental imperfections: losses during transfer of the atom between the two traps and during illumination of the atom in the dipole trap, imperfect state initialization by optical pumping, and erroneous detection of the atomic state. Fig. 3 also shows that the measured spectra are in very good agreement with the theoretical prediction from a numerical Bloch-
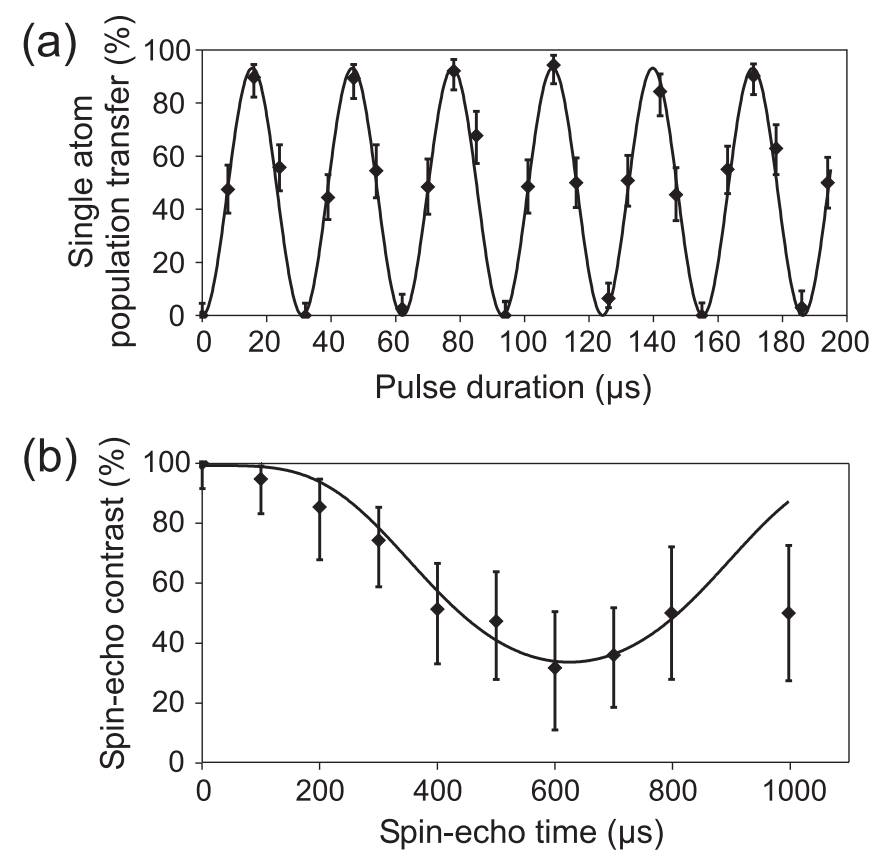

FIG. 4: Coherent qubit manipulation. (a) Demonstration of qubit rotations of individually addressed single atoms. Each point shows the averaged population transfer of approximately 40 single atom events. The Rabi-oscillations have a contrast of $99.1_{-3.7}^{+0.9} \%$. (b) Contrast of the spin-echo signal of individually addressed atoms as a function of the spin-echo time. The decrease of the spin-echo contrast to $35 \%$ after $600 \mu \mathrm{s}$ is caused by radial oscillations of the atoms in an inhomogeneous magnetic field. The fit from a theoretical model (solid line) is in good agreement with the measured data.

vector simulation with no adjusted parameters. The same simulation also allows us to calculate the coherent shift $\Delta \varphi$ of the relative phase between states $|0\rangle$ and $|1\rangle$ induced in adjacent atoms due to non-resonant interaction with the detuned microwave pulse. For the experimental parameters of Fig. 3 (c) and an atom separation of e. g. $2.5 \mu \mathrm{m}$, this calculation yields a phase shift $\Delta \varphi=0.2 \cdot \pi$. It can be taken into account in further gate operations.

Arbitrary qubit rotations of our quantum register are demonstrated in Fig. 4 (a). Here, we trap, image, and initialize one atom qubit as above. After the application of a square microwave pulse of duration $t_{\text {pulse }}$ at the corresponding resonance frequency of the atom, we measure the transfer probability to state $|1\rangle$. Every point in Fig. 4 (a) corresponds to the transfer probability deduced from approximately 40 single atom events. The signal shows Rabi oscillations of the population between states $|0\rangle$ and $|1\rangle$. This state evolution reads $\left|\psi\left(t_{\text {pulse }}\right)\right\rangle=\cos \left(\Omega_{R} t_{\text {pulse }} / 2\right)|0\rangle-i \sin \left(\Omega_{R} t_{\text {pulse }} / 2\right)|1\rangle$, where $\Omega_{R}=2 \pi \cdot 32 \mathrm{kHz}$ is the Rabi frequency. A pulse duration of $\tau=\pi / 2 \Omega_{R}=8 \mu$ s therefore corresponds to a one qubit Hadamard-gate in quantum information pro- 
cessing. The line in Fig. 4 (a) is a sinusoidal fit which yields a contrast of $99.1_{-3.7}^{+0.9} \%$. We hereby demonstrate reliable single qubit rotations on our quantum register.

In order to investigate the coherence properties of the quantum register we have performed a spin-echo measurement [13] on single atoms addressed in the magnetic field gradient. The spin-echo contrast is shown in Fig. 4 (b) as a function of the spin-echo time. We measure a reduction of the contrast to approximately $35 \%$ within $600 \mu \mathrm{s}$. Note that this dephasing time is two orders of magnitude larger than the measured one-qubit switching time. A possible two-qubit gate performed by the exchange of single photons in an optical high-finesse cavity has an expected operation time of 200 ns and would be more than three orders of magnitude faster than the dephasing time of our quantum register [17].

The solid line in Fig. 4 (b) is a theoretical fit which models the effect of the thermal oscillations of the trapped atoms inside the applied inhomogeneous magnetic field. According to equation (11) we see that the modulus of the magnetic field in radial direction varies as

$$
|\vec{B}(x=0, y, z)| \approx B_{0}+\frac{1}{2 B_{0}} B^{\prime 2}\left(4 z^{2}+y^{2}\right) .
$$

The Zeeman shift of the $|1\rangle \leftrightarrow|0\rangle$ transition therefore depends on the radial position of the atoms. Consequently, a radial oscillation will result in a time varying atomic resonance frequency and cause a dephasing between states $|0\rangle$ and $|1\rangle$. Due to the oscillatory behaviour of this dephasing, the resulting reduction of the spin-echo contrast varies periodically. Complete rephasing is theoretically possible for a spin-echo time of twice the radial oscillation period. However, the maximum time interval in which quantum operations can continuously be performed is determined by the initial decay of the spin-echo contrast.

We find very good agreement between our model and the experimental data for typical experimental parameters (see Fig. 4 (b)): a temperature of the atomic ensemble of $80 \mu \mathrm{K}$, a radial oscillation frequency of $1.6 \mathrm{kHz}$, and the dipole trap axis running $15 \mu \mathrm{m}$ above or below the symmetry plane of the B-field due to alignment imperfections. This result indicates that the initial reduction of our spin-echo contrast is predominantly caused by the thermal radial oscillations of the atoms in our trap. Possible ways to extend the coherence time of our quantum register therefore include an increase of the magnetic offset field $B_{0}$ (see Eq. (2)), a more advantageous magnetic field geometry, a reduction of the radial oscillations by further cooling of the atoms, and a transfer of the atoms to decoherence-free states between addressing operations.

Summarizing our results, we have demonstrated that a string of caesium atoms trapped in our standing wave dipole trap can be used as a quantum register. We have initialized, selectively addressed, coherently manipulated, and state-selectively detected the hyperfine states of individual atoms within the string. Our scheme operates on atoms separated by distances as small as $2.5 \mu \mathrm{m}$. Therefore, if the qubits were evenly spaced, populating every fifth trapping site, it is scalable to a few hundred qubits, limited by the axial extension of the trapping potential.

Currently, we set up a second, perpendicular conveyorbelt which should allow us to place each individual atom of the quantum register into a desired potential well. This would enable us to distribute the atoms evenly in the trapping region. Furthermore, it should permit to induce controlled interaction of arbitrary pairs of distant atom qubits by placing them next to each other. Our scheme is compatible with the requirements of cavity quantum electrodynamics or controlled cold collision experiments, making our quantum register a versatile tool for the implementation of quantum logic operations.

We thank W. Alt and S. Kuhr for valuable discussions and technical assistance. This work has been supported by the Deutsche Forschungsgemeinschaft and the European Commission.

* Electronic address: meschede@iap.uni-bonn.de

[1] P. Shor, SIAM J. Comp. 26, 1484 (1997).

[2] L. K. Grover, Phys. Rev. Lett. 79, 325 (1997).

[3] S. Lloyd, Phys. Rev. Lett. 75, 346 (1995).

[4] R. Raussendorf and H. J. Briegel, Phys. Rev. Lett. 86, 5188 (2001).

[5] H. C. Nägerl et al., Phys. Rev. A 60, 145 (1999).

[6] F. Schmidt-Kaler et al., Nature 422, 408 (2003).

[7] D. Leibfried et al., Nature 422, 412 (2003).

[8] L. M. K. Vandersypen et al., Nature 414, 883 (2001).

[9] T. Yamamoto et al., Nature 425, 941 (2003).

[10] S. Kuhr et al., Science 293, 278 (2001). [published online; 10.1126/science.1062725].

[11] N. Schlosser, G. Reymond, I. Protsenko, and P. Grangier, Nature 411, 1024 (2001).

[12] N. Davidson et al., Phys. Rev. Lett. 74, 1311 (1995).

[13] S. Kuhr et al., Phys. Rev. Lett. 91, 213002 (2003).

[14] O. Mandel et al., Nature 425, 937 (2003).

[15] S. Osnaghi et al., Phys. Rev. Lett. 87, 037902 (2001).

[16] T. Pellizzari, S. A. Gardiner, J. I. Cirac, and P. Zoller, Phys. Rev. Lett. 75, 3788 (1995).

[17] L. You, X. X. Yi, and X. H. Su, Phys. Rev. A 67, 032308 (2003).

[18] D. Schrader et al., Appl. Phys. B 73, 819 (2001).

[19] W. Alt, Optik 113, 142 (2002).

[20] Y. Miroshnychenko et al., Optics Express 11, 3498 (2003).

[21] D. Haubrich et al., Europhys. Lett. 34, 663 (1996).

[22] W. Alt et al., Phys. Rev. A 67, 033403 (2003). 